\title{
HELIUM IN THE UNIVERSE: FINAL SUMMARY
}

\author{
G. BURBIDGE \\ University of California, San Diego, Calif., U.S.A. \\ and \\ Institute of Theoretical Astronomy, Cambridge University, Cambridge, U.K.
}

It is my task to summarize for you what we have learned today about the helium problem. Before attempting this, let me first very briefly outline the problem as it appears to us in 1970. It amounts to the questions: What is the fractional abundance of helium in different regions in the universe, is it the same in all places, and how did it originate?

The simple answers to these questions which appealed to many and seemed to have a reasonable basis in fact a few years ago were:

$\mathrm{The} \mathrm{He} / \mathrm{H}$ ratio is of $\sim 25-30 \%$ by mass; the ratio is the same everywhere, and the helium was most probably synthesized in the early stages of a primordial fireball in which the universe began. However, for some time it has been clear that the situation is more complicated than this, as I pointed out in an article published last year in Comm. Astrophys. Space Sci.

Today we heard first from Dr Cayrel who summarized the information presently available on the helium abundance in stars. He discussed both the disk Population I B stars and the halo stars. In the bulk of the well-studied B stars, studies using model atmospheres give results which suggest that the $\mathrm{He} / \mathrm{H}$ ratio is $\sim 25 \%$. At the same time, it appears that the possibility of departures from local thermodynamic equilibrium can introduce some uncertainties. According to Cayrel, these are likely to be rather small, while Dr Underhill once again in the discussion expressed the opinion that they could be large. The most important question is centred about the B stars with very weak helium lines which have recently been discussed in detail by Norris. Analyses of these lead to the conclusion that the $\mathrm{He} / \mathrm{H}$ ratio is very small. This is a very important result since it may mean that the material out of which these stars condensed had a very small helium abundance. The uncertainties in reaching this conclusion are twofold. Do very weak helium lines in the stars' atmospheres mean that the helium abundance is very low, and is it possible that the composition of the atmospheres is not the same as that in the deeper layers of the stars? It has been suggested that the abundance determination is uncertain because of possible departures from LTE. I consider this unlikely. The other possibility - that gravitational separation is important - also seems very improbable since all of the other elements have normal abundance.

Cayrel also reviewed the data on the compositions of the halo-type (Population II) stars. It is well known that some of these stars have exceedingly weak helium lines; in some cases there is no evidence for any helium at all, and the work of Sargent 
and Searle and Greenstein and Münch suggested that the helium abundance might be very low in these stars. However, doubt was cast on this conclusion when Sargent and Searle found other spectroscopic anomalies in some stars of this type, which led them to believe that abundance anomalies might be due to the nuclear evolution of the stars and did not necessarily reflect the composition of the gas out of which the stars condensed. One Population II object which has a normal (high) helium abundance is the planetary nebula in M15. (This was in fact discussed by Seaton.) In the discussion Schwarzschild suggested that this might very well be largely the helium abundance of the gas out of which the stars in the cluster condensed, rather than helium made in the star and then ejected when the planetary formed. Of course there is no certainty about this, but it is a possibility.

Seaton reviewed the determinations of the $\mathrm{He} / \mathrm{H}$ ration in gaseous nebulae, both in our Galaxy and elsewhere. He discussed some aspects of the methods of obtaining abundances and the uncertainties involved. The nebulae in our own Galaxy all do show the 'normal' $\approx 25 \%$ figure with some uncertainties which may be due either to uncertainties in observation and analysis, or to small differences. The value for planetary nebulae is about $26 \%$ and this is consistent with the idea that the stars condensed out of gas with a normal $\mathrm{He} / \mathrm{H}$ ratio and that there has been a small enrichment of the helium content due to thermonuclear burning of hydrogen. The fragmentary data on the $\mathrm{He} / \mathrm{H}$ ratio in external galaxies - mostly comparatively closeby - tend to give values of $\approx 25 \%$ with some scatter. In M31 Rubin and Ford have investigated ratios of line intensities in gaseous nebulae over a wide range of distances from the center. They have found that there are considerable variations in the $\mathrm{He} / \mathrm{H}$ ratio from place to place, though there is no systematic variation as a function of distance from the center. This is a particularly important result.

Dr Faulkner discussed the problems associated with deriving a helium abundance from theoretical arguments of stellar structure. He discussed first the tangled problem associated with the mass-luminosity relation derived by Eggen, and the Hyades. He concluded that the large helium abundances derived by Eggen are probably not correct. Perhaps all that one can say at present is that $Y_{\text {Hyades }} \lesssim 1.5 Y_{\odot}$ with $Y_{\text {Hyades }} \approx Y_{\odot}$ not excluded. In the discussion Dr Popper showed that one obtains a normal helium abundance from well-observed binaries.

Faulkner pointed out that, when one attempts to fit evolutionary tracks in the giant branches with observation, a better fit is obtained for high $Y(\geqslant 0.25)$ than for low $Y(\leqslant 0.10)$. For horizontal branches it also appears that $Y \geqslant 0.25$. He pointed out that there are still considerable uncertainties when one comes to the models for the RR Lyrae stars.

A basic uncertainty which is coming more and more to haunt us again in attempts to derive ages or chemical composition of stars, using theoretical stellar models, is the problem of the opacities, and until this is cleared up we cannot expect to get very far. There are still considerable problems associated with self-consistent models for the sun.

Dr Kippenhahn considered the question of how much helium can be made as a 
star evolves and how it might be got out. He found that this is a very difficult business. Helium which is made is very easily destroyed. In the case of very massive stars, pulsational instability might eject considerable amounts of helium, but not enough to account for the bulk of the helium present.

We then turned to the problem of the production of helium on the cosmological scale. Dr Novikov gave an account of present ideas concerning the production of helium in the early stages of a big bang universe. The important point that he stressed, as has also Dr Fowler in a talk to Commission 47 and in a paper appearing in Comm. Astrophys. Space Sci., is that there is no particular reason to believe that, even if there was a big bang, a universal helium/hydrogen ratio $\sim 25-30 \%$ was generated. Neither the temperature of the black body radiation from a big bang nor the abundance of helium synthesized in a big bang is determined by first principles of general relativity. To obtain the temperature and the helium abundance, it is necessary to specify the baryon number, the electron lepton number, and the muon lepton number. Values of the helium abundance ranging from 0 to $100 \%$ can be obtained by suitable choices of these quantities. Thus for $n_{v} / n_{\gamma}=1$, where $n_{v}$ is the number density of electron neutrinos and $n_{\gamma}$ is the number density of photons, the helium abundance produced in a big bang is less than $1 \%$.

Finally, Dr Wagoner reviewed investigations concerned with helium production in supermassive stars and disks which may be responsible for helium production in the nuclei of galaxies. He showed that, while uniformly rotating massive stars can convert pure hydrogen, or hydrogen with a normal composition of carbon, nitrogen, or oxygen, into helium, they will only be able to eject a significant helium fraction if the mass loss time due to pulsational instability is comparable with the nuclear burning time, and this is unlikely. Uniformly rotating supermassive disks $(M \lesssim 2 \times$ $\times 10^{10}-3 \times 10^{9} M_{\odot}$, depending on the initial $\mathrm{C}, \mathrm{N}$, or $\mathrm{O}$ content) can also produce helium. If the disk does not fragment, a nuclear explosion may ensue as the disk evolves. Little bangs, in which it is just assumed that matter in an initial high density state with initial temperatures $\sim 10^{10} \mathrm{deg} \mathrm{K}$ expands at rates somewhat different from the gravitational rate, can make helium in amounts ranging from $\sim 10-70 \%$ of hydrogen.

What have we now learned from these discussions? There may be a uniform abundance of helium in all objects, but I doubt it. In particular, the results for the B stars, and those for M31, cast doubt on this hypothesis. If there are wide variations in the $\mathrm{He} / \mathrm{H}$ ratio, then the bulk of the helium was not formed in a big bang. Perhaps there never was one, despite the tremendous need by so many to believe in the Old Testament. Big bang or not, the bulk of the helium may have been made in the nuclear regions of galaxies. It should be pointed out that the very large fluxes of infrared radiation which are now being discovered in some galaxies may alter completely our ideas concerning the total bolometric luminosities of galaxies. If these fluxes have a thermonuclear origin, the old discrepancy between the total energy radiated, based on optical luminosities, and the amount of helium seen in the bulk of the Population I objects, may be removed. 
The origin of helium in the universe, a problem which was known to be complex, in part because we had little knowledge ten or fifteen years ago, and which was then thought by many to be resolved following the discovery of the microwave radiation five years ago, is now seen clearly to be even more complicated than we originally thought it to be. As usual, one offers the pious hope that more observations will improve the situation. Perhaps this is so, but the problem is likely to be with us for a long time yet. 